Received: 9 May 2018

Accepted: 9 May 2018

Published online: 21 May 2018

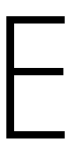

N
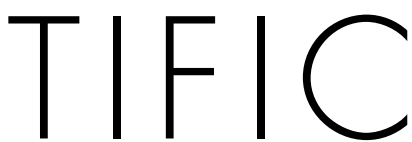

RE

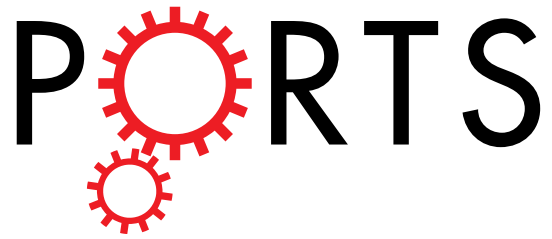

OPEN

\section{The live cell DNA stain SiR-Hoechst induces DNA damage responses and impairs cell cycle progression}

\author{
Onur Sen ${ }^{1}$, Adrian T. Saurin $\mathbb{1}^{2}$ \& Jonathan M. G. Higgins ${ }^{1}$
}

SiR-Hoechst (SiR-DNA) is a far-red fluorescent DNA probe being used widely for time-lapse imaging of living cells that is reported to be minimally toxic at concentrations as high as $10-25 \mu \mathrm{M}$. However, measuring nuclear import of Cyclin B1, inhibition of mitotic entry, and the induction of $\gamma \mathrm{H} 2 \mathrm{AX}$ foci in cultured human cells reveals that SiR-Hoechst induces DNA damage responses and G2 arrest at concentrations well below $1 \mu \mathrm{M}$. SiR-Hoechst is useful for live cell imaging, but it should be used with caution and at the lowest practicable concentration.

The ability to observe chromatin in living cells is invaluable in cell biology, allowing individual cells to be followed within cultures or tissues, and the fate of chromosomes within cells to be tracked (for example during cell division or apoptosis). Cell permeable fluorescent DNA dyes that allow chromatin to be visualized in many cell types without the need for introducing exogenous fluorescent proteins by transfection are therefore appealing. However, DNA dyes such as Hoechst 33342 are known to cause DNA damage, particularly during DNA replication, and so alter the behaviour of the cells under observation. Such damage may be brought about by disruption of cellular processes because of binding of the dye to DNA, by photochemical toxicity caused by excitation of the fluorescent molecule, or by a combination of the two ${ }^{1-3}$. A recently developed cell-permeable DNA probe, SiR-Hoechst (also known as $\mathrm{SiR}-\mathrm{DNA})^{4}$, is reported not to cause toxicity and has been commercialized, widely publicized, and adopted by numerous laboratories for live cell imaging ${ }^{5-37}$. SiR-Hoechst has some clear advantages: it is selective for DNA; its fluorescence is enhanced upon DNA binding; it is excited by far-red light, avoiding damage caused by the UV light required for traditional Hoechst dyes; and it is compatible with live-cell super-resolution microscopy. However, although in the original report there was little detectable effect on mitotic progression (over $3.4 \mathrm{~h}$ ) or proliferation of transformed HeLa cells (over $24 \mathrm{~h}$ ), no detailed analyses of cell cycle progression or specific measurements of DNA damage were carried out in either transformed or in non-transformed cell lines ${ }^{4}$.

\section{Results and Discussion}

During a normal cell cycle, Cyclin B1 accumulates in the cytoplasm and at centrosomes during G2, enters the nucleus several minutes before nuclear envelope breakdown at the onset of mitosis, and then is degraded during mitotic exit ${ }^{38,39}$. In transformed cell lines such as U2OS, DNA damage prevents the nuclear import of Cyclin B1 and cells arrest in G2 with high levels of cytoplasmic Cyclin B1 ${ }^{40-42}$. By contrast, in non-transformed cell lines such as hTert-immortalized RPE1, Cyclin B1 is imported into the nucleus in a p21-dependent manner during G2 in response to DNA damage, and accumulation of Cyclin B1 at centrosomes remains low ${ }^{41-45}$. Hours later, Cyclin $\mathrm{B} 1$ is degraded in the absence of mitosis, and the cells become senescent ${ }^{41,42,45}$.

To track Cyclin B1 localisation in response to SiR-Hoechst, we used RPE1 and U2OS cell lines that express Cyclin B1-EYFP from its endogenous locus ${ }^{46,47}$. We treated RPE1 and U2OS cells with a range of SiR-Hoechst concentrations ${ }^{4}$, and observed the localisation of both Cyclin B1-EYFP and SiR-Hoechst by live imaging for 18 to $19 \mathrm{~h}$. In RPE1 cells we observed two major cell fates: (i) timely Cyclin B1 import prior to mitosis, and (ii) Cyclin B1 import followed by later degradation in the absence of mitosis, reflecting arrest in G2 (Fig. 1a). Among control cells treated with DMSO that imported Cyclin B1 into the nucleus, 3\% displayed non-mitotic import of Cyclin B1 (see example Supplemental Movie 1), but this was significantly increased to $24 \%$ in cells treated with $1 \mu \mathrm{M}$ SiR- Hoechst (Supplemental Movie 2, Fig. 1c). An increase in the percentage of RPE1 cells showing non-mitotic import of Cyclin B1 was also seen at $0.5 \mu \mathrm{M}$ and $0.25 \mu \mathrm{M}$ SiR-Hoechst, though the magnitude of this

${ }^{1}$ Cell Division Biology Group, Institute for Cell and Molecular Biosciences, Newcastle University, Medical School, Framlington Place, Newcastle upon Tyne, NE2 4HH, UK. ${ }^{2}$ Division of Cancer Research, School of Medicine, Jacqui Wood Cancer Centre, Ninewells Hospital and Medical School, University of Dundee, Dundee, DD1 9SY, UK. Correspondence and requests for materials should be addressed to J.M.G.H. (email: jonathan.higgins@ncl.ac.uk) 
a

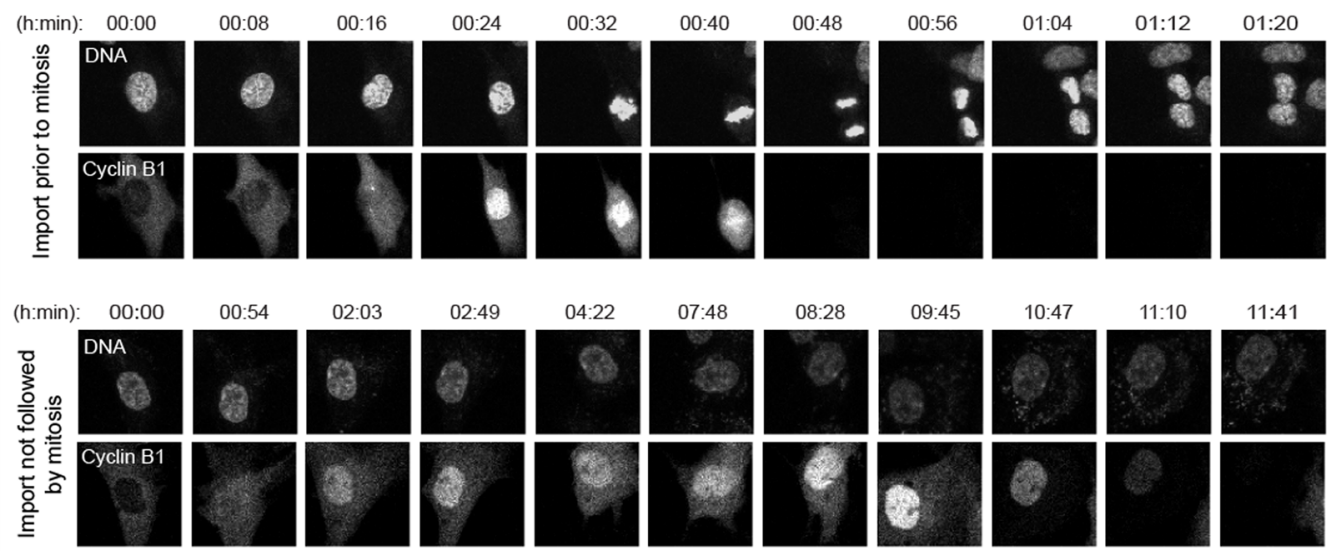

b

$($ himin): $00: 00$
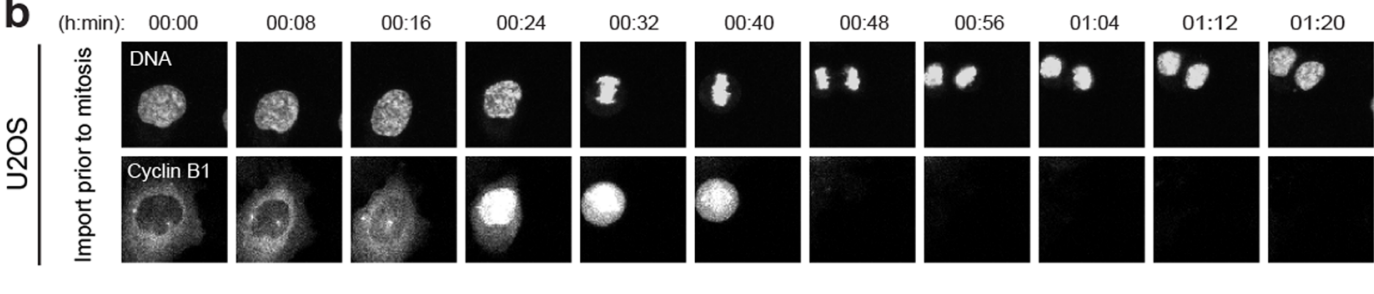

C

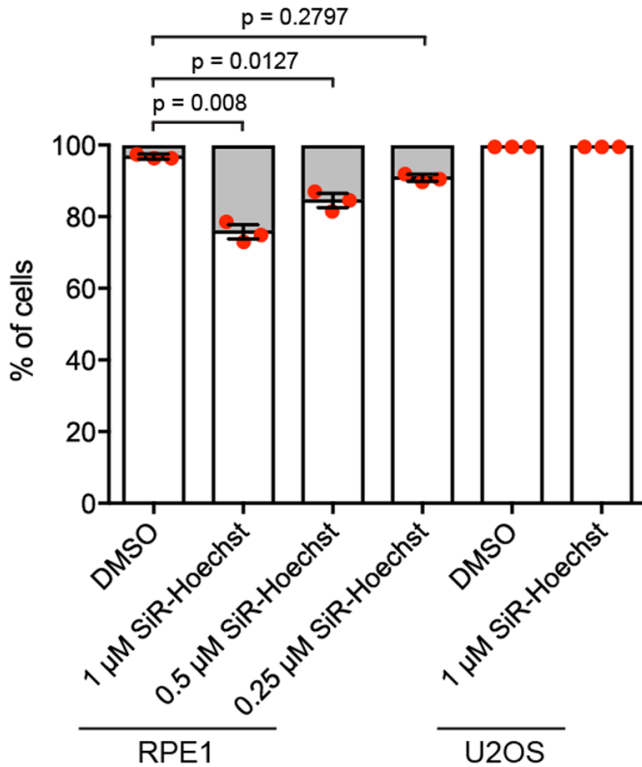

Cyclin B1 import not followed by mitosis

Cyclin B1 import prior to mitosis

Figure 1. Live imaging in the presence of SiR-Hoechst causes nuclear retention of Cyclin B1 in RPE1 cells, independent of mitosis. (a) Asynchronous RPE1 cells expressing Cyclin B1-EYFP were treated with DMSO or different concentrations $(1 \mu \mathrm{M}, 0.5 \mu \mathrm{M}, 0.25 \mu \mathrm{M})$ of SiR-Hoechst for $2 \mathrm{~h}$ prior to live imaging for $18-19 \mathrm{~h}$ in the continued presence of DMSO or SiR-Hoechst. Representative movie stills illustrate the two cell fates observed. Also see Supplemental Movies 1 to 4. (b) Asynchronous U2OS cells expressing Cyclin B1-EYFP were treated with DMSO or $1 \mu \mathrm{M}$ SiR-Hoechst for $2 \mathrm{~h}$ prior to live imaging for $18 \mathrm{~h}$ in the continued presence of SiR-Hoechst. In addition, all U2OS cells were treated with $10 \mu \mathrm{M}$ Verapamil to inhibit efflux pumps. Representative movie stills illustrate the cell fate observed. Also see Supplemental Movies 5 and 6. (c) For cells showing nuclear import of Cyclin B1, the fates indicated in (a,b) were quantified in three independent experiments. Between 152 and 623 cells were counted per treatment. Error bars indicate mean \pm SEM $(n=3)$. Statistical significance was determined using unpaired t-tests.

effect declined as the concentration was decreased (Fig. 1c; Supplemental Movies 3 and 4). As expected, the transformed cell line U2OS did not display non-mitotic nuclear import of Cyclin B1, in either controls or after treatment with $1 \mu \mathrm{M}$ SiR-Hoechst, but Cyclin B1 accumulated in the cytoplasm over longer periods in the presence of SiR-Hoechst (Fig. 1b,c; Supplemental Movies 5 and 6). Therefore, both RPE1 and U2OS cells show evidence of an arrest or delay in G2 in response to SiR-Hoechst. 

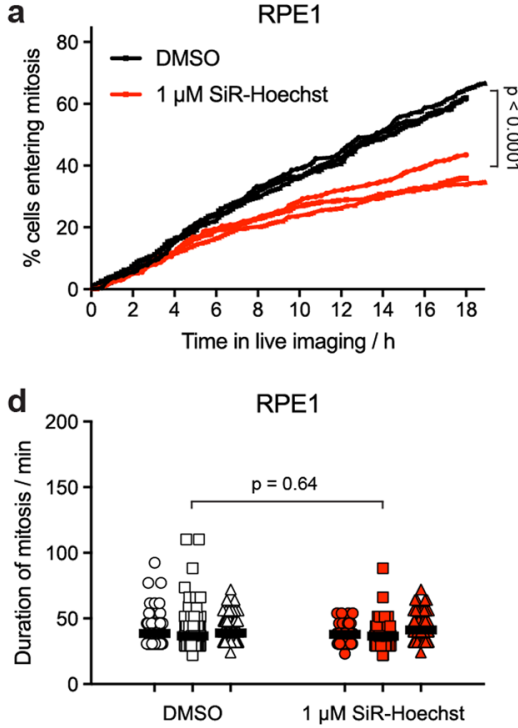

b
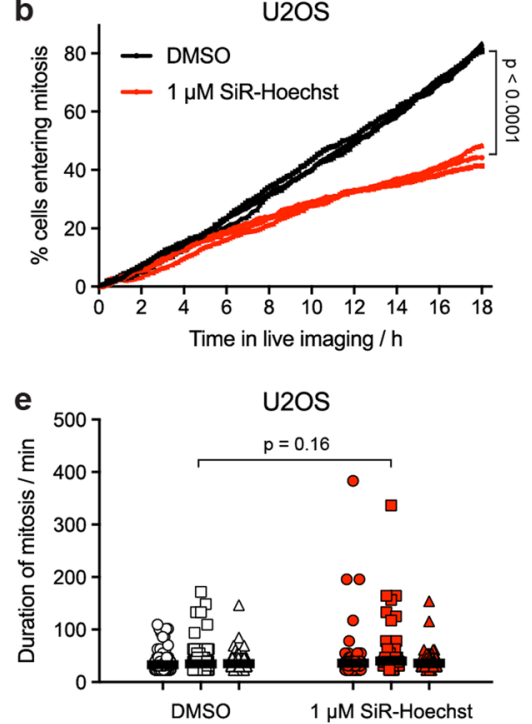

C

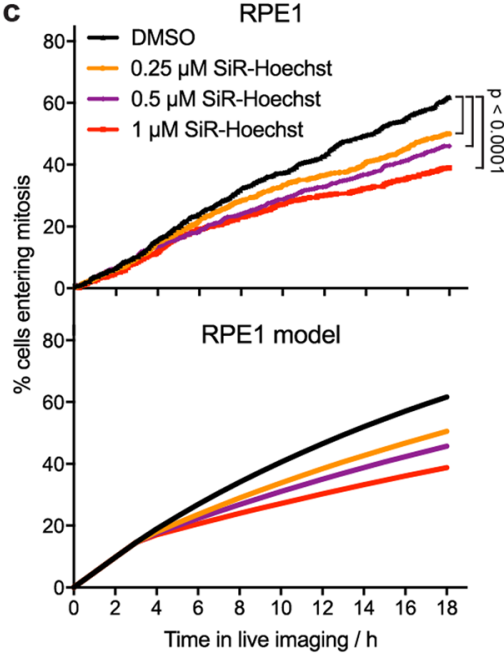

Figure 2. Live imaging in the presence of SiR-Hoechst delays cell division but does not alter the duration of mitosis. (a) Asynchronous RPE1 cells expressing Cyclin B1-EYFP were treated with DMSO or $1 \mu \mathrm{M}$ SiRHoechst for $2 \mathrm{~h}$ then imaged for 18-19h in the continued presence of DMSO or SiR-Hoechst. Mitotic entry was defined as the first live imaging frame in which mitotic nuclear import of Cyclin B1 was detected. Between 265 and 453 total cells were counted per treatment. The results of three independent experiments are shown. Statistical significance was determined from the combined data using Kaplan-Meier curve analysis and a log rank test. (b) As for (a), but for U2OS cells expressing Cyclin B1-EYFP. Between 561 and 695 total cells were counted per treatment $(n=3)$. (c) Top panel. As for (a), but RPE1 cells expressing Cyclin B1-EYFP were treated with DMSO, $0.25,0.5$ or $1 \mu \mathrm{M}$ SiR-Hoechst. The combined results of 4 fields of view from two independent experiments are shown. Between 689 and 889 total cells were counted per treatment. Bottom panel. Theoretical time courses of RPE1 cell mitotic entry derived from the mathematical model using the following parameters: normal length of cell cycle, $20.6 \mathrm{~h}$ (derived from Supplemental Fig. 2); length of G2 plus M, $5.2 \mathrm{~h}^{49}$; imaging damage coefficient, 0.6; pre-incubation time in SiR-Hoechst, $2 \mathrm{~h}$; percentage of cells arresting in G2 (determined from imaging of non-mitotic G2 import) in DMSO, 2.1\%; in $0.25 \mu \mathrm{M}$ SiR-Hoechst, $6.9 \%$; in $0.5 \mu \mathrm{M}$ SiR-Hoechst, 7.4\%; in $1 \mu \mathrm{M}$ SiR-Hoechst, 9.9\%. (d) The duration of mitosis for the RPE1 cells quantified in (a) was defined as described in Methods. Between 114 to 266 mitotic cells were quantified per treatment, in three independent experiments. Horizontal bars indicate the mean duration of mitosis. Statistical significance was determined from the combined data using a paired t-test $(n=3)$. (e) As for $(d)$, but for U2OS cells expressing Cyclin B1EYFP. Between 227 and 544 mitotic cells were quantified per treatment $(n=3)$.

As an alternative way to monitor cell cycle delay in both RPE1 and U2OS cells, we counted the number of cells entering mitosis during live imaging. The presence of $1 \mu \mathrm{M}$ SiR-Hoechst caused the number of RPE1 and U2OS cells entering mitosis to drop by more than $40 \%$ over the period of imaging (Fig. 2a,b). This outcome was concentration-dependent, but declines in mitotic entry were also observed at 0.5 and $0.25 \mu \mathrm{M}$ SiR-Hoechst (Fig. 2c). To estimate the extent of the delay, we developed a simple mathematical model of cell cycle progression in which SiR-Hoechst caused DNA damage in S phase, leading to either cell cycle arrest or delay in S/G2. For RPE1, cells arresting in G2 were defined as those that imported Cyclin B1 in the absence of mitosis during live imaging (approximately $10 \%$ of all cells at $1 \mu \mathrm{M}$ SiR-Hoechst). The rates of mitotic entry observed at $0.25,0.5$ and $1 \mu \mathrm{M}$ SiR-Hoechst were best modelled when the remaining non-arresting cells were delayed in S/G2 for an average of 8, 14 or 26 hours, respectively (Fig. 2c; Supplemental Fig. 1a). For U2OS cells, which do not import Cyclin B1 into the nucleus in response to DNA damage, the model suggested that $\mathrm{S} / \mathrm{G} 2$ was extended by an average of $33 \mathrm{~h}$ at $1 \mu \mathrm{M}$ SiR-Hoechst (Supplemental Fig. 1b). In both the results and the models, the effect of SiR-Hoechst was most pronounced after $4 \mathrm{~h}$ or more of live imaging (Fig. 2a-c; Supplemental Fig. 1), reflecting the ability of cells that are already in G2 when SiR-Hoechst is added to proceed relatively unimpeded into mitosis. Reducing the intensity of laser illumination during imaging did not prevent the detrimental effect of SiR-Hoechst on RPE1 cells (Supplemental Fig. 2). Although cells in mitosis itself have sometimes been considered particularly sensitive to damage during live imaging ${ }^{4,48}$, for those cells that divided, SiR-Hoechst had little influence on the duration of mitosis in either RPE1 or U2OS cells (Fig. 2d,e). These findings show that, during live imaging, SiR-Hoechst can prevent a significant portion of cells from entering mitosis, and suggest that it induces a DNA damage response that delays or arrests cell cycle progression in G2. In addition, they demonstrate that normal mitotic duration cannot be taken as evidence that cells are unaffected by the dye.

To determine if this effect of SiR-Hoechst on living RPE1 cells requires light exposure during microscopy, we conducted experiments in which cells were treated with SiR-Hoechst for $20 \mathrm{~h}$ in the absence of imaging, followed by fixation and immunofluorescence microscopy. Among cells that had nuclear Cyclin B1, we then determined the proportion that was in G2 or mitosis. Cells with uncondensed chromatin and nuclear but not centrosomal Cyclin B1, and typically with nuclear p21, were classified as in G2. Cells with nuclear Cyclin B1 and 
a

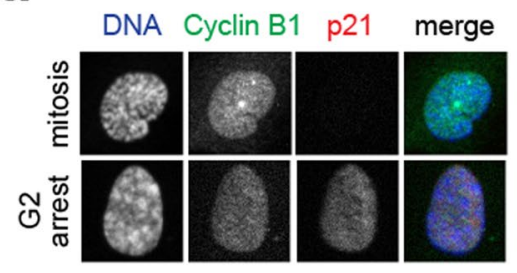

C

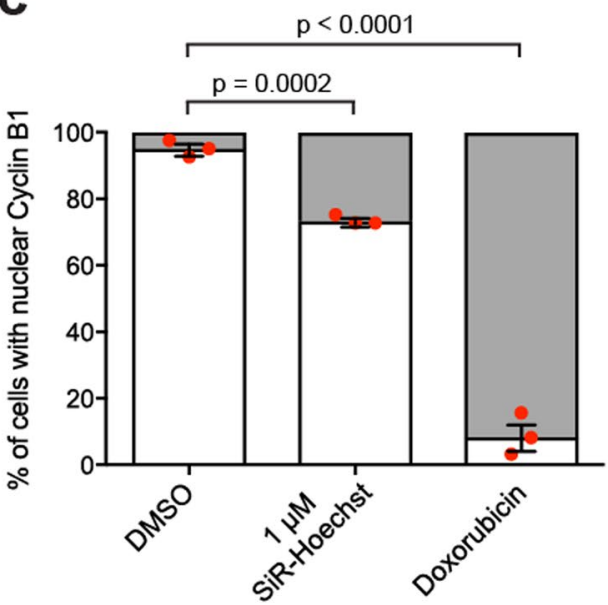

b

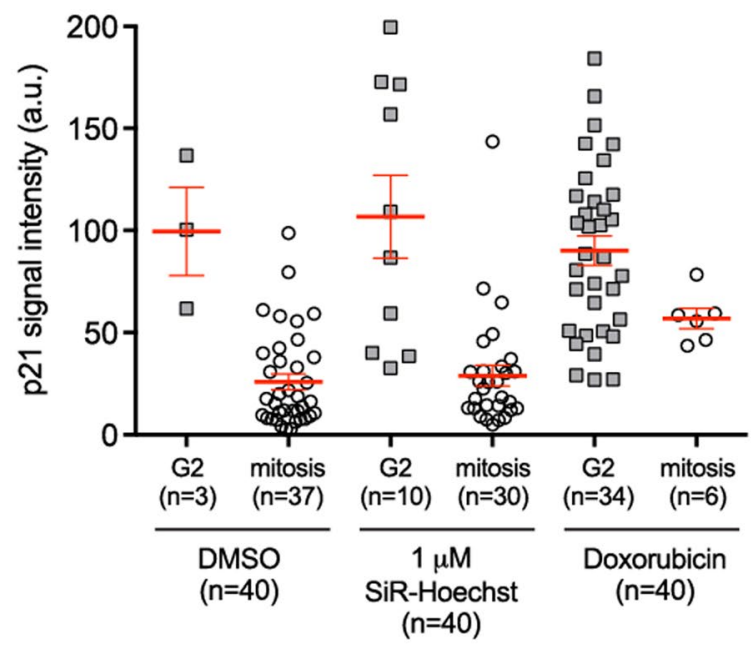

Figure 3. SiR-Hoechst causes non-mitotic nuclear Cyclin B1 import in the absence of imaging. (a) Asynchronous RPE1 cells expressing Cyclin B1-EYFP were treated with DMSO, $1 \mu \mathrm{M}$ SiR-Hoechst or $0.5 \mu \mathrm{M}$ Doxorubicin for $20 \mathrm{~h}$ before fixation. Fixed cells were co-stained with Hoechst 33342 (DNA), anti-GFP (Cyclin B1-EYFP) and anti-p21 antibodies. Representative images show G2 or mitotic cells that imported Cyclin B1 into nucleus. (b) Asynchronous RPE1 cells expressing Cyclin B1-EYFP were treated and stained as in (a). Cells with nuclear Cyclin B1 were classified as in G2 (cells with uncondensed nuclear chromatin and without centrosomal Cyclin B1) or in mitosis (cells with clearly condensing chromosomes and/or centrosomal Cyclin B1), and the intensity of nuclear p21 staining was quantified in 40 cells per treatment in one experiment. Means \pm SEM are shown. Note that cells classified as arrested in G2 typically have higher levels of p21 than cells entering mitosis. (c) The cell fates indicated in $(\mathrm{a}, \mathrm{b})$ were quantified in 40 cells per treatment in three independent experiments. Error bars indicate mean $\pm \operatorname{SEM}(\mathrm{n}=3)$. Statistical significance was determined using unpaired $\mathrm{t}$-tests.

clearly condensing chromosomes and/or centrosomal Cyclin B1, typically without nuclear p21, were classified as in mitosis (see Fig. 3a,b). Among control DMSO-treated cells with nuclear Cyclin B1, 5\% were in G2, while the majority (95\%) were in prophase of mitosis (Fig. 3c). When treated with the known DNA damaging agent Doxorubicin, the majority (92\%) of cells with nuclear Cyclin B1 were in G2. Notably, $1 \mu \mathrm{M}$ SiR-DNA treatment also caused a significant increase, to $27 \%$, in the percentage of cells with nuclear Cyclin B1 that were in G2 (Fig. 3c). These data show that binding of SiR-Hoechst to DNA is sufficient to cause nuclear Cyclin B1 retention in G2 in the absence of imaging, although laser exposure may exacerbate the effect (see Supplemental Fig. 2).

Finally, we measured the induction of the DNA damage marker $\gamma \mathrm{H} 2 \mathrm{AX}$ (H2AX S139ph) to determine if SiR-Hoechst caused DNA damage in cells. As expected, Doxorubicin strongly induced $\gamma \mathrm{H} 2 \mathrm{AX}$ in both RPE1 and U2OS cell lines (Fig. 4a,b). At $1 \mu \mathrm{M}$, SiR-Hoechst caused a small increase in $\gamma \mathrm{H} 2 \mathrm{AX}$ foci in both RPE1 and U2OS cells and, at $5 \mu \mathrm{M}$, more clearly induced $\gamma \mathrm{H} 2 \mathrm{AX}$ foci (Fig. 4a,b) particularly in RPE1 cells. Therefore, SiR-Hoechst may cause DNA damage even in the absence of imaging.

By the measures of non-mitotic nuclear import of Cyclin B1, inhibition of mitotic entry and the induction of $\gamma \mathrm{H} 2 \mathrm{AX}$ foci, it is clear that SiR-Hoechst causes changes in cell cycle progression, probably at least in part due to DNA damage. U2OS cells, as well as the widely-used non-transformed cell line RPE1, are susceptible to a substantial G2 delay or arrest, even well below the manufacturer's recommended concentration of $1 \mu \mathrm{M}$. A majority of published studies use SiR-Hoechst at a concentration of $0.5 \mu \mathrm{M}$ or higher (Supplemental Table 1), but we find that it is not a benign DNA stain in living cells. Our results show that concentrations below $0.25 \mu \mathrm{M}$ should be used to minimize cell cycle progression defects. These concentrations are sufficient for high-quality imaging, at least by laser scanning confocal microscopy. Importantly, because the duration of mitosis can be unaffected under conditions in which the rate of mitotic entry is substantially reduced, apparently normal chromosome congression and segregation cannot be taken as evidence that SiR-Hoechst is not altering the biology of the cells under observation. We hope that the observations and simple mathematical model reported here will help others to optimize suitable conditions for their own experiments. SiR-Hoechst is an excellent addition to the cell biologist's toolbox, but we urge researchers to use it at the lowest possible concentration. 
a

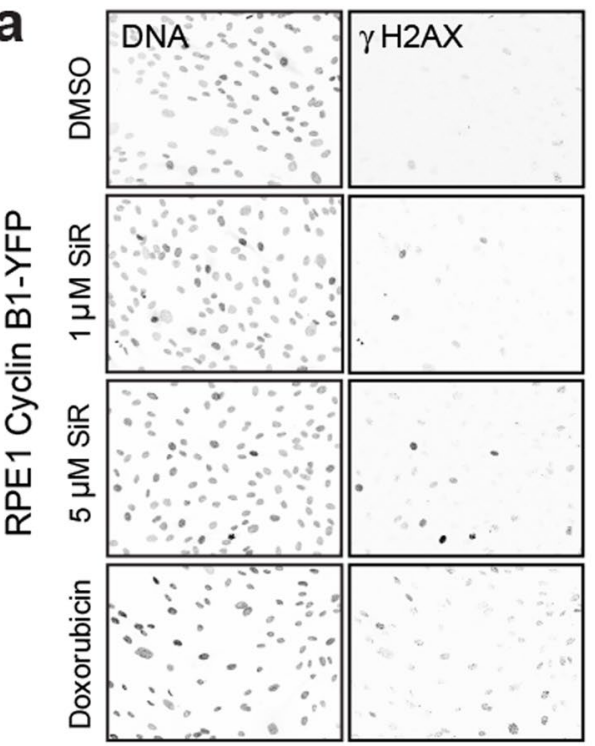

b

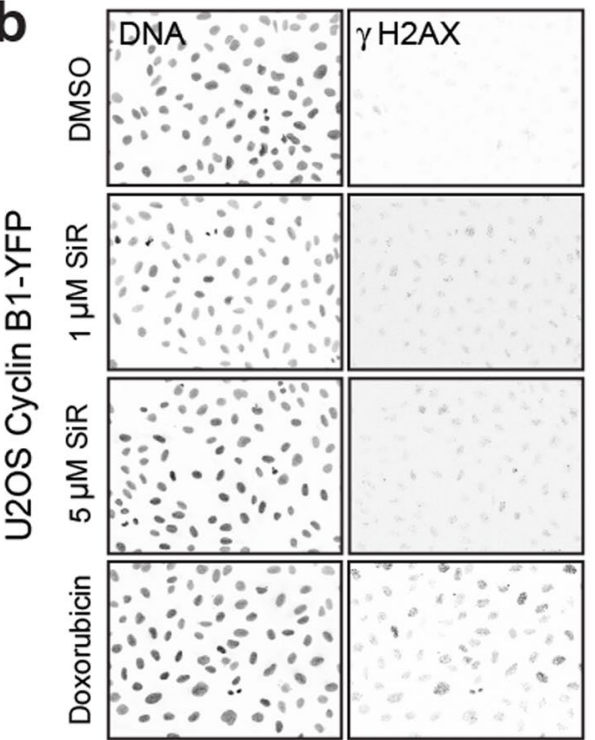

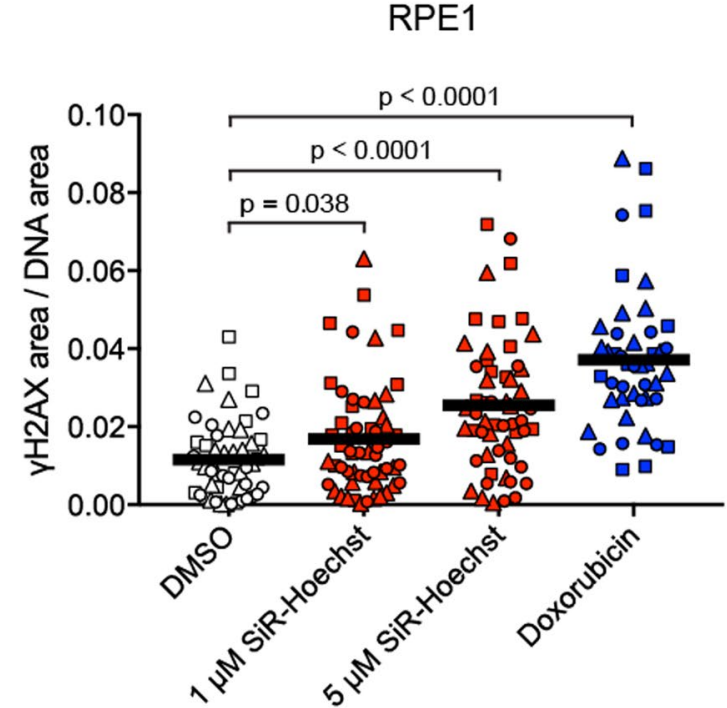

\section{U2OS}

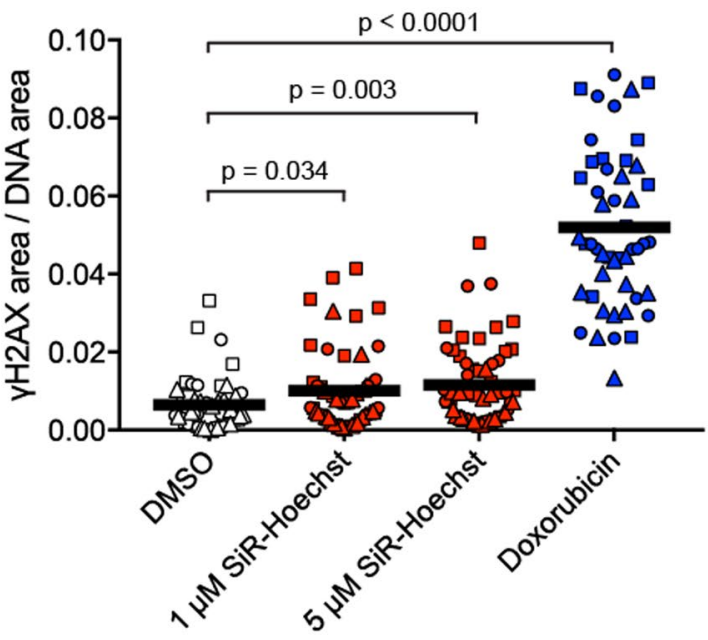

Figure 4. SiR-Hoechst can cause DNA damage in the absence of imaging. (a) Asynchronous RPE1 cells expressing Cyclin B1-EYFP were treated with DMSO, 1 or $5 \mu \mathrm{M}$ SiR-Hoechst, or $0.5 \mu \mathrm{M}$ Doxorubicin for $20 \mathrm{~h}$, followed by fixation. Fixed cells were co-stained with Hoechst 33342 (DNA) and anti- $\gamma \mathrm{H} 2 \mathrm{AX}$ antibodies. The proportion of pixels that exceeded a fixed threshold of $\gamma \mathrm{H} 2 \mathrm{AX}$ intensity was then determined in three independent experiments (circle, square, triangle symbols) and reported as the (area containing $\gamma \mathrm{H} 2 \mathrm{AX}$ staining)/(area containing DNA staining, i.e. nuclei). Approximately 18 fields were measured per condition in each experiment. Means are indicated by horizontal bars. Statistical significance was determined using unpaired $\mathrm{t}$-tests ( $\mathrm{n}=44$ to 56). (b) Asynchronous U2OS cells expressing Cyclin B1-EYFP were treated and analysed as in (a). Statistical significance was determined using unpaired t-tests $(n=49$ to 58$)$. In addition, U2OS cells were treated with $10 \mu \mathrm{M}$ Verapamil throughout to inhibit efflux pumps.

\section{Methods}

Cell culture. U2OS or hTert-RPE1 cells that express Cyclin B1-EYFP from its endogenous locus ${ }^{46,47}$ were cultured in DMEM or DMEM/F12 media (Sigma), respectively, supplemented with 10\% Foetal Bovine Serum (FBS) (Labtech) and penicillin/streptomycin (Sigma) at $37^{\circ} \mathrm{C}$ and $5 \% \mathrm{CO}_{2}$.

Time-lapse microscopy. Cells were seeded in 4-chamber glass-bottom dishes (Greiner Bio One) $24 \mathrm{~h}$ before imaging. Two hours before imaging, cells were loaded with SiR-Hoechst (SiR-DNA; Spirochrome) or vehicle control (DMSO). In addition, U2OS cells were treated with $10 \mu \mathrm{M}$ Verapamil (Spirochrome) to inhibit dye efflux pathways and enable homogenous DNA staining ${ }^{4}$. Time-lapse imaging was then carried out at $37^{\circ} \mathrm{C}, 5 \% \mathrm{CO}_{2}$ for 18 to 18.9 hours using an inverted Nikon A1R confocal microscope. Observations were performed throughout using a Plan Apo 20x 0.75 NA air objective with an excitation pixel dwell time of $2.4 \mu$ s. Images of $1024 \times 1024$ 
pixels were captured with a pixel size of $0.63 \mu \mathrm{m}$. Confocal pinholes were set to capture approximately $2 \mu \mathrm{m}$ thick optical sections for each channel. Sections were taken every $5 \mu \mathrm{m}$ over $20 \mu \mathrm{m}$ ( 5 sections in total). Volumes were captured approximately every 8 minutes. Channel observation conditions were as follows. For EYFP: excitation wavelength $514 \mathrm{~nm}$; laser power recorded at object of $47 \mu \mathrm{W}$; GaSaP detector gain of 43/255. For SiR-Hoechst: excitation wavelength $640 \mathrm{~nm}$; laser power recorded at object of $190 \mu \mathrm{W}$; standard PMT detector gain of 81/255. Where stated, alternative settings for SiR-Hoechst were used: excitation wavelength $640 \mathrm{~nm}$; laser power recorded at object of $86 \mu \mathrm{W}$; standard PMT detector gain of $81 / 255$. Power measurements were made in the same imaging conditions using a Thorlabs S170c microscope slide photodiode sensor and PM100D meter. All images were manually analysed and quantified using NIS-Elements (Nikon) software. The time of mitotic entry was determined as the first movie frame in which Cyclin B1-EYFP rapidly entered the nucleus prior to cell division visualised by transmitted light imaging. The duration of mitosis was defined as the time elapsed between this frame and the first frame in which Cyclin B1-EYFP was eliminated in anaphase. Statistical analyses were carried out using Prism 7 (GraphPad Software).

Cell Cycle Model. To model the effect of SiR-Hoechst on the cell cycle, we assumed that dye-induced DNA damage is caused during DNA replication (S phase) and that this results in cell cycle arrest or delay in S or G2 phases. In the model, cells that are in G2 or mitosis at the time of SiR-Hoechst addition can proceed unimpeded into and through mitosis (first term in the equation below). Cells that pass through $\mathrm{S}$ phase during imaging can have one of three fates. Two populations suffer delays in S/G2 of $t_{x}$ or $t_{y}$ hours (where $t_{x}$ or $t_{y} \geq 0$, and $t_{x}$ or $t_{y}=\infty$ when arrested), represented by the second and third terms in the equation. Another population is unaffected by SiR-Hoechst (final term in equation). Other parameters are as follows: $t_{c}$ is the normal duration of one cell cycle (determined using imaging conditions in which \% mitotic entry increased linearly for the entire duration of imaging); $t_{G 2 M}$ is the normal length of time spent in G2 and M phases (obtained from the literature) ${ }^{46,49} ; t_{p}$ is the time in hours during which SiR-Hoechst was pre-incubated with cells prior to imaging; $t_{i}$ is the number of hours since imaging began; $c$ is a coefficient accounting for progressive damage caused by imaging in the absence of SiR-Hoechst; $x$ and $y$ are the fractions of cells suffering a delay of $\mathrm{t}_{\mathrm{x}}$ and $\mathrm{t}_{\mathrm{y}}$ hours, respectively (where $x+y \leq 1$ ).

$$
\begin{aligned}
\% \text { cells entering mitosis }= & \frac{100\left(\min \left(\max \left(t_{G 2 M}-t_{p}, 0\right), t_{i}\right)\right)}{t_{c}} \\
& +\frac{100 x\left(t_{i}-\max \left(t_{G 2 M}-t_{p}, 0\right), 0\right)}{t_{c}+t_{x}+c t_{i}} \\
& +\frac{100 y\left(t_{i}-\max \left(t_{G 2 M}-t_{p}, 0\right), 0\right)}{t_{c}+t_{y}+c t_{i}} \\
& +\frac{100(1-x-y)\left(t_{i}-\max \left(t_{G 2 M}-t_{p}, 0\right), 0\right)}{t_{c}+c t_{i}}
\end{aligned}
$$

Immunofluorescence microscopy. Cells were seeded on glass coverslips coated with poly-lysine and, after $24 \mathrm{~h}$, treated with SiR-Hoechst (SiR-DNA; Spirochrome), doxorubicin hydrochloride (Sigma) or DMSO for $20 \mathrm{~h}$ before fixation with $2 \%$ (w/v) paraformaldehyde (Thermo Fisher Scientific) in PBS for 10 minutes at room temperature. After washing three times with PBS, cells were incubated in $0.5 \%(\mathrm{v} / \mathrm{v})$ Triton X-100 in PBS for 10 minutes, washed three times with PBS and blocked in BlockAid solution (Thermo Fisher Scientific) for $1 \mathrm{~h}$ at room temperature. Cells were stained for $18 \mathrm{~h}$ at $4{ }^{\circ} \mathrm{C}$ with the following primary antibodies diluted in BlockAid solution: mouse anti-GFP monoclonal antibody (Abcam, ab1218), mouse anti-phospho-Histone H2AX Ser-139 $(\gamma \mathrm{H} 2 \mathrm{AX}$ ) monoclonal antibody (Merck Millipore, 05-636), rabbit anti-p21 polyclonal antibody (Abcam, ab109520). After washing three times with $0.05 \%(\mathrm{v} / \mathrm{v})$ Triton X-100 in PBS, cells were stained with the following secondary antibodies diluted in BlockAid solution for $1 \mathrm{~h}$ at $37^{\circ} \mathrm{C}$ : goat anti-rabbit Alexa Fluor 488 or Alexa Fluor 594, donkey anti-mouse Alexa Fluor 488 or Alexa Fluor 594 (Jackson ImmunoResearch). After washing, cells were stained with $2 \mu \mathrm{g} / \mathrm{ml}$ Hoechst 33342 (Thermo Fisher Scientific, H2570) in PBS for 5 min at room temperature, before washing with PBS and mounting using Prolong Diamond Antifade Mountant (Thermo Fisher Scientific, P36961). Images were obtained using a Zeiss Axio Imager, equipped with Apotome, 20x 0.8 NA air objective, LED light source, AxioCam MR R3 camera and ZEN (Zeiss) software. To obtain $\gamma \mathrm{H} 2 \mathrm{AX} / \mathrm{DNA}$ signals, images of approximately 18 fields per treatment in each experiment on a coverslip were captured. DNA and $\gamma \mathrm{H} 2 \mathrm{AX}$ signals were quantified using Photoshop CS6 by determining the number of pixels within each image exceeding a fixed threshold intensity for $\gamma \mathrm{H} 2 \mathrm{AX}$ signal ("fuzziness" level 80) and DNA signal (level 40). The signal was then expressed as a ratio of the area containing $\gamma \mathrm{H} 2 \mathrm{AX}$ staining over the area containing DNA signal. The signal intensity of nuclear p21 was quantified in G2 and prophase cells using ImageJ. Statistical analyses were carried out using Minitab 17 (Minitab Inc.) and Prism 7 (GraphPad Software).

\section{References}

1. Durand, R. E. \& Olive, P. L. Cytotoxicity, Mutagenicity and DNA damage by Hoechst 33342. J. Histochem. Cytochem. 30, 111-116 (1982).

2. Smith, P. J. \& Anderson, C. O. Modification of the radiation sensitivity of human tumour cells by a bis-benzimidazole derivative. Int. J. Radiat. Biol. 46, 331-344 (1984).

3. Purschke, M., Rubio, N., Held, K. D. \& Redmond, R. W. Phototoxicity of Hoechst 33342 in time-lapse fluorescence microscopy. Photochem. Photobiol. Sci. 9, 1634-1639 (2010).

4. Lukinavicius, G. et al. SiR-Hoechst is a far-red DNA stain for live-cell nanoscopy. Nat. Commun. 6, 8497 (2015). 
5. Anton, T. \& Bultmann, S. Site-specific recruitment of epigenetic factors with a modular CRISPR/Cas system. Nucleus 8, 279-286 (2017).

6. Cerboni, S. et al. Intrinsic antiproliferative activity of the innate sensor STING in T lymphocytes. J. Exp. Med. 214, 1769-1785 (2017).

7. Chittori, S. et al. Structural mechanisms of centromeric nucleosome recognition by the kinetochore protein CENP-N. Science 359, 339-343 (2018).

8. Cuylen, S. et al. Ki-67 acts as a biological surfactant to disperse mitotic chromosomes. Nature 535, 308-312 (2016).

9. Elbatsh, A. M. O. et al. Condensin's ATPase Machinery Drives and Dampens Mitotic Chromosome Condensation. bioRxiv, 216630 (2017).

10. Fava, L. L. et al. The PIDDosome activates 553 in response to supernumerary centrosomes. Genes Dev. 31, 34-45 (2017).

11. Fiorentino, F. P., Bagella, L. \& Marchesi, I. A new parameter of growth inhibition for cell proliferation assays. J. Cell. Physiol. 233, 4106-4115 (2018).

12. Fourriere, L., Divoux, S., Roceri, M., Perez, F. \& Boncompain, G. Microtubule-independent secretion requires functional maturation of Golgi elements. J. Cell Sci. 129, 3238-3250 (2016).

13. Hilbert, L. et al. Transcription establishes microenvironments that organize euchromatin. bioRxiv, 234112 (2017).

14. Karakashev, S. et al. BET Bromodomain Inhibition Synergizes with PARP Inhibitor in Epithelial Ovarian Cancer. Cell Rep. 21, 3398-3405 (2017).

15. Kubitschke, H. et al. Actin and microtubule networks contribute differently to cell response for small and large strains. New J. Phys. 19, 093003 (2017).

16. Kukolj, E. et al. PARP inhibition causes premature loss of cohesion in cancer cells. Oncotarget 8, 103931-103951 (2017).

17. Leyton-Puig, D. et al. Flat clathrin lattices are dynamic actin-controlled hubs for clathrin-mediated endocytosis and signalling of specific receptors. Nat. Commun. 8, 16068 (2017).

18. Li, Y. et al. Structural basis of the phosphorylation-independent recognition of cyclin D1 by the SCF(FBXO31) ubiquitin ligase. Proc. Natl Acad. Sci. USA 115, 319-324 (2018).

19. Libouban, M. A. A. et al. Stable aneuploid tumors cells are more sensitive to TTK inhibition than chromosomally unstable cell lines. Oncotarget 8, 38309-38325 (2017).

20. McKinley, K. L., Stuurman, N., Klein, O. D. \& Vale, R. D. The actin cytoskeleton governs apical mitosis and daughter cell dispersion in intestinal epithelia. bioRxiv, 183301 (2017).

21. Mehedi, M. et al. Actin-Related Protein 2 (ARP2) and Virus-Induced Filopodia Facilitate Human Respiratory Syncytial Virus Spread. PLoS Pathog. 12, e1006062 (2016).

22. Natsume, T., Kiyomitsu, T., Saga, Y. \& Kanemaki, M. T. Rapid Protein Depletion in Human Cells by Auxin-Inducible Degron Tagging with Short Homology Donors. Cell Rep. 15, 210-218 (2016).

23. Novak, M. et al. The mitotic spindle is chiral due to torques generated by motor proteins. bioRxiv, 167437 (2017).

24. Otsuka, S. et al. Nuclear pore assembly proceeds by an inside-out extrusion of the nuclear envelope. eLife 5, e19071 (2016).

25. Rochman, M., Travers, J., Abonia, J. P., Caldwell, J. M. \& Rothenberg, M. E. Synaptopodin is upregulated by IL-13 in eosinophilic esophagitis and regulates esophageal epithelial cell motility and barrier integrity. JCI Insight 2, e96789 (2017).

26. Samejima, K. et al. Rapid degradation of condensins and 3D-EM reveal chromatin volume is uncoupled from chromosome architecture in mitosis. J. Cell Sci. 131, jcs210187 (2018).

27. Shaban, H. A., Barth, R. \& Bystricky, K. Formation of correlated chromatin domains at nanoscale dynamic resolution during transcription. Nucleic Acids Res. https://doi.org/10.1093/nar/gky296 (2018).

28. Silio, V., Millar, J. B. A. \& McAinsh, A. D. Cell transformation disrupts the efficiency of chromosome segregation through microtubule detyrosination. bioRxiv, 246983 (2018).

29. Smith, L. et al. The responses of cancer cells to PLK1 inhibitors reveal a novel protective role for $\mathrm{p} 53$ in maintaining centrosome separation. Sci. Rep. 7, 16115 (2017).

30. Stojic, L. et al. Specificity of RNAi, LNA and CRISPRi as loss-of-function methods in transcriptional analysis. bioRxiv, 234930 (2017).

31. Vorobjev, I. A. \& Barteneva, N. S. Multi-parametric imaging of cell heterogeneity in apoptosis analysis. Methods 112, 105-123 (2017).

32. Walther, N. et al. A quantitative map of human Condensins provides new insights into mitotic chromosome architecture. J. Cell Biol. https://doi.org/10.1083/jcb.201801048 (2018).

33. Wutz, G. et al. Topologically associating domains and chromatin loops depend on cohesin and are regulated by CTCF, WAPL, and PDS5 proteins. EMBO J. 36, 3573-3599 (2017).

34. Yost, S. et al. Biallelic TRIP13 mutations predispose to Wilms tumor and chromosome missegregation. Nat. Genet. 49, 1148-1151 (2017).

35. Yuan, R. et al. Chk1 and 14-3-3 proteins inhibit atypical E2Fs to prevent a permanent cell cycle arrest. EMBO J. 37, e97877 (2018).

36. Zierhut, C. \& Funabiki, H. The cytoplasmic DNA sensor cGAS promotes mitotic cell death. bioRxiv, 168070 (2017).

37. Currie, C. E., Mora-Santos, M. D. M., Smith, C., McAinsh, A. D. \& Millar, J. B. A. Bub1 is not required for the checkpoint response to unattached kinetochores in diploid human cells. bioRxiv, 278820 (2018).

38. Pines, J. \& Hunter, T. Human cyclins A and B1 are differentially located in the cell and undergo cell cycle-dependent nuclear transport. J. Cell Biol. 115, 1-17 (1991).

39. Hagting, A., Jackman, M., Simpson, K. \& Pines, J. Translocation of cyclin B1 to the nucleus at prophase requires a phosphorylationdependent nuclear import signal. Curr. Biol. 9, 680-689 (1999).

40. Winters, Z. E., Ongkeko, W. M., Harris, A. L. \& Norbury, C. J. p53 regulates Cdc2 independently of inhibitory phosphorylation to reinforce radiation-induced G2 arrest in human cells. Oncogene 17, 673-684 (1998).

41. Krenning, L., Feringa, F. M., Shaltiel, I. A., van den Berg, J. \& Medema, R. H. Transient activation of p53 in G2 phase is sufficient to induce senescence. Mol. Cell 55, 59-72 (2014).

42. Mullers, E., Silva Cascales, H., Jaiswal, H., Saurin, A. T. \& Lindqvist, A. Nuclear translocation of Cyclin B1 marks the restriction point for terminal cell cycle exit in G2 phase. Cell Cycle 13, 2733-2743 (2014).

43. Porter, L. A., Cukier, I. H. \& Lee, J. M. Nuclear localization of cyclin B1 regulates DNA damage-induced apoptosis. Blood 101, 1928-1933 (2003).

44. Charrier-Savournin, F. B. et al. p21-Mediated nuclear retention of cyclin B1-Cdk1 in response to genotoxic stress. Mol. Biol. Cell 15, 3965-3976 (2004).

45. Lossaint, G., Besnard, E., Fisher, D., Piette, J. \& Dulic, V. Chk1 is dispensable for G2 arrest in response to sustained DNA damage when the ATM/p53/p21 pathway is functional. Oncogene 30, 4261-4274 (2011).

46. Akopyan, K. et al. Assessing kinetics from fixed cells reveals activation of the mitotic entry network at the S/G2 transition. Mol. Cell 53, 843-853 (2014).

47. Shaltiel, I. A. et al. Distinct phosphatases antagonize the $\mathrm{p} 53$ response in different phases of the cell cycle. Proc. Natl Acad. Sci. USA 111, 7313-7318 (2014).

48. Johnson, I. D. Practical Considerations in the Selection and Application of Fluorescent Probes. In Handbook of Biological Confocal Microscopy (ed. Pawley, J.) 353-367 (Springer Science \& Business Media, 2006).

49. McKinley, K. L. \& Cheeseman, I. M. Large-Scale Analysis of CRISPR/Cas9 Cell-Cycle Knockouts Reveals the Diversity of p53Dependent Responses to Cell-Cycle Defects. Dev Cell 40, 405-420 e402 (2017). 


\section{Acknowledgements}

We are very grateful to Drs Alex Laude, Glyn Nelson and Rolando Berlinguer Palmini of the Newcastle University Bio-Imaging Facility for their help. This study was funded by a Wellcome Trust Investigator Award and a Royal Society Wolfson Research Merit Award to J.M.G.H. and an award from Cancer Research UK (C47320/A21229) to A.T.S.

\section{Author Contributions}

O.S. planned, conducted and analysed experiments and co-wrote the manuscript. A.T.S. provided the cell lines, suggested experiments and made comments on the manuscript. J.M.G.H. was responsible for direction of the project, planned and analysed experiments, developed the mathematical model, and co-wrote the manuscript.

\section{Additional Information}

Supplementary information accompanies this paper at https://doi.org/10.1038/s41598-018-26307-6.

Competing Interests: The authors declare no competing interests.

Publisher's note: Springer Nature remains neutral with regard to jurisdictional claims in published maps and institutional affiliations.

(c) (i) Open Access This article is licensed under a Creative Commons Attribution 4.0 International License, which permits use, sharing, adaptation, distribution and reproduction in any medium or format, as long as you give appropriate credit to the original author(s) and the source, provide a link to the Creative Commons license, and indicate if changes were made. The images or other third party material in this article are included in the article's Creative Commons license, unless indicated otherwise in a credit line to the material. If material is not included in the article's Creative Commons license and your intended use is not permitted by statutory regulation or exceeds the permitted use, you will need to obtain permission directly from the copyright holder. To view a copy of this license, visit http://creativecommons.org/licenses/by/4.0/.

(C) The Author(s) 2018 